\title{
Modeling of Joule heating and convective cooling in a thick-walled micro-tube
}

\author{
Kevin D. Cole ${ }^{\mathrm{a}}$, Barbaros Çetin ${ }^{\mathrm{b}, *}$ \\ ${ }^{a}$ Mechanical Engineering Department, N104 Scott Engineering Center, University of Nebraska-Lincoln, 68588-0656 Lincoln, NB, USA \\ ${ }^{\mathrm{b}}$ Microfluidics and Lab-on-a-chip Research Group, Mechanical Engineering Department, İ.D. Bilkent University, 06800 Ankara, Turkey
}

\section{A R T I C L E I N F O}

\section{Article history:}

Received 27 November 2016

Received in revised form

4 April 2017

Accepted 11 May 2017

Available online 29 May 2017

\section{Keywords:}

Micro-channel heat transfer

Joule heating

Graetz problem

Green's function

\begin{abstract}
A B S T R A C T
The heating of a fluid in a metallic micro-tube can be realized at the inlet and/or within a certain section of micro-scale heat and fluid flow devices by using Joule heating which is a heat generation mechanism that occurs when an electric current is passed through the metallic wall. For the thermal analysis of fluid flow in an electrically heated micro-tube, the solution of conjugate heat transfer (to include effect of the axial conduction through the channel wall) together with Joule heating is required. An analytic solution is presented for conjugate heat transfer in an electrically-heated micro-tube in this study. The solution is obtained in the form of integrals by the method of Green's functions for the hydrodynamically fullydeveloped flow of a constant property fluid in a micro-tube. The current analytical model can predict the fluid temperature for a given wall thickness, wall material and applied voltage across the micro-tube. The effects of the wall thickness and the wall material on the normalized temperature distribution and the effectiveness parameter are discussed. The comparison of the normalized temperature for Joule heating and a spatially uniform heating is also presented.
\end{abstract}

() 2017 Elsevier Masson SAS. All rights reserved.

\section{Introduction}

Micro-channels are the key component for many micro-scale fluid, heat and mass flow devices such as micro-heat-exchangers, micro-heat-sinks, micro-reactors and microfluidic fuel cells [1]. As the device scale moves to the micro-scale, some effects which can be safely neglected at the macro-scale have to be considered [2]. As a result of these effects, the classical fluid flow and heat transfer theories, correlations and design methodology may not be suitable at micro-scale. Additional effects depend on the working fluid (i.e. whether the flow is a gas or liquid flow), channel size as well as the channel material. Rarefaction and compressibility is an important parameter for gas flows even for low Mach number flows $(M a \sim 0.1)$ [3]. Depending on the degree of rarefaction, slip-flow and temperature-jump boundary condition together with thermal creep might need to be included in the analysis [4-6]. Viscous dissipation [7] and axial conduction within the fluid [8] may be quite important in the case of liquid flow. Moreover, in the case of liquid flow, the effect of the relative roughness [9] and the electroviscous effects (if the channel wall is non-conducting) [10] may

\footnotetext{
* Corresponding author.

E-mail address: barbaros.cetin@bilkent.edu.tr (B. Çetin).
}

come into picture.

Although the channel wall thickness is usually small compared to the channel size for macro-channels, the thickness of the channel wall is often comparable to the channel size due to rigidity and fabrication concerns. Furthermore, although the convective heat transfer is the dominant heat transfer mode for macro-channels, the heat conduction through the channel walls and within the fluid becomes comparable with the fluid convection due to the low Peclet number nature of the heat transfer for micro-channels. Nowadays, micro-tubes made of copper, nickel, aluminum and stainless steel with an inner diameter of 100-900 $\mu \mathrm{m}$ are commercially available with different choice of connectors to build a fluidic network (the size data of some of the commercially available micro-tubes is given in Table 1). Typically, the wall thickness of micro-tubes is ranging between 25 and $700 \mu \mathrm{m}$. Especially for the small diameter micro-tubes, the wall thickness may be much larger than the inner diameter of the tube. Therefore, the heat transferred within the channel wall needs to be taken into account for the calculation of the overall heat transfer.

The conjugate heat transfer problem, which refers to the solution of convective heat transfer in a conduit together with heat conduction through conduit wall, has been studied by many researchers [11]. The conjugate heat transfer for macro-channels was 


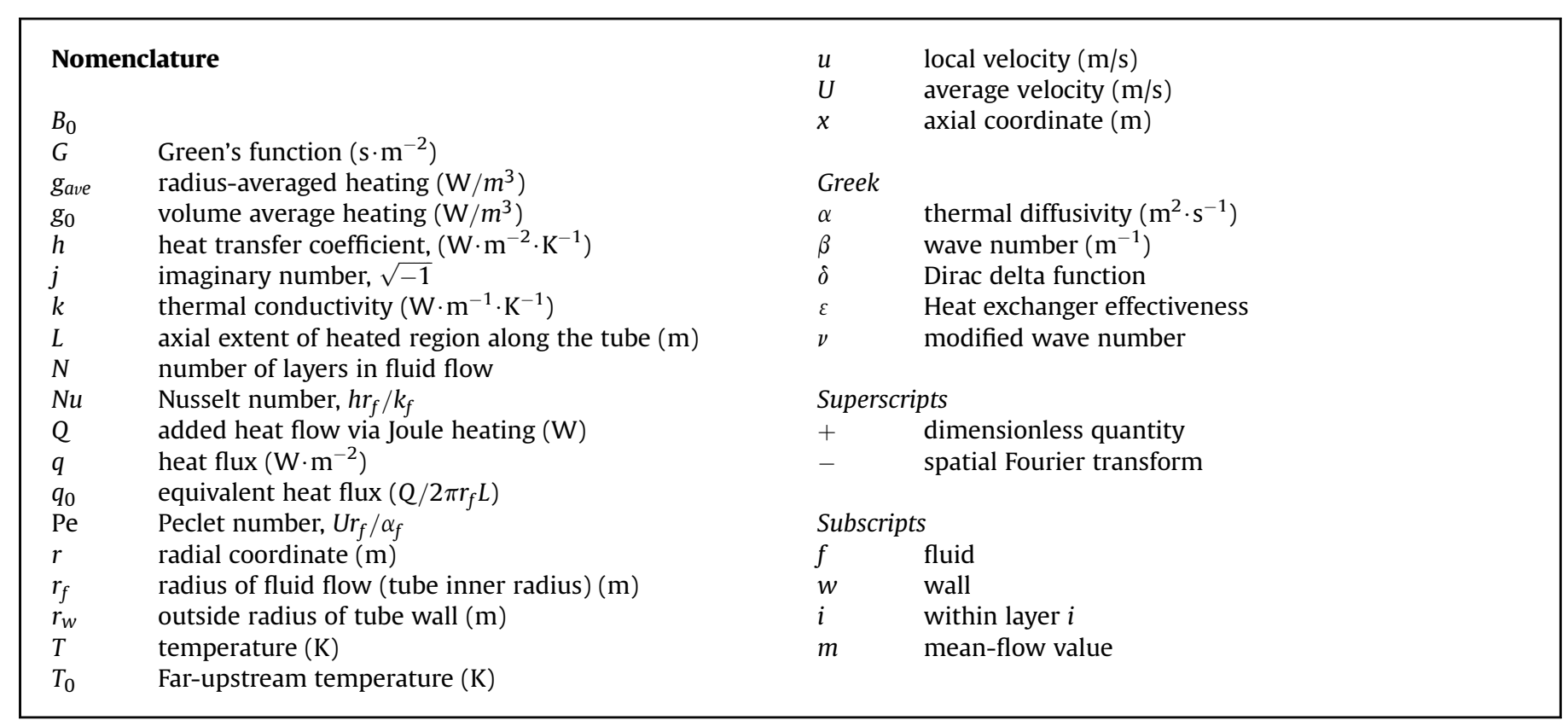

Table 1

Some typical values for the size and material of the microtubes.

\begin{tabular}{lllll}
\hline Reference & Material & $\mathrm{d}(\mu \mathrm{m})$ & $\mathrm{D}(\mu \mathrm{m})$ & Wall thickness $(\mu \mathrm{m})$ \\
\hline$[25,26]$ & Stainless steel & 170 & 1588 & 709 \\
{$[25,26]$} & Stainless steel & 510 & 1588 & 539 \\
{$[25,26]$} & Stainless steel & 750 & 1588 & 419 \\
{$[27]$} & Nickel & 200 & 780 & 290 \\
{$[27]$} & Nickel & 300 & 350 & 25 \\
{$[27]$} & Nickel & 350 & 600 & 125 \\
{$[27]$} & Nickel & 500 & 800 & 150 \\
\hline
\end{tabular}

studied couple decades ago with the exclusion of the axial conduction within the fluid [12,13]. More recently, parallel to the development in the micro-fabrication techniques, the conjugate heat transfer for micro-channels was investigated for different geometries such as circular [14-16], parallel-plate [17-20], rectangular [21-23] and converging-diverging [24]. In these studies, the axial conduction within the fluid is also considered due to the low Peclet number nature of the flow. Inclusion of axial heat conduction in the channel wall and in the fluid introduce some challenges in the solution. One challenge is the coupling of the heat conduction problem with the heat convection problem associated with the inclusion of the axial heat conduction in the wall. A second challenge is the non-self adjoint eigen-value nature of the problem if a solution is an analytical technique based-on eigen-function expansion associated with the inclusion of the heat conduction within the fluid [8]. Many researchers implemented numerical solution to overcome these drawbacks [14-16,20-22,24]. Alternatively, many researchers proposed different analytical techniques which offers a fast and highly accurate solution, namely infinite Fourier transform $[18,19]$ and general integral transform technique [23].

The heating of a fluid in a micro-tube can be desirable at the inlet and/or within a certain section of micro-scale heat and fluid flow devices. For macro-tubes, the convenient way to supply heat to the channel wall is to wrap an electric-resistance heater around a tube which realize a constant heat flux thermal boundary condition at the tube boundary. However, in the case of metallic micro-tubes, the convenient way to supply heat to the channel wall is to use Joule heating, which is a heat generation mechanism that occurs when an electric current is passed through the metallic wall. In this case, the heat flux at the solid-fluid interface is a result of the volumetric heat generation which takes place within the channel wall. Several researchers utilized electrical heating of a tube for the experimental investigation of the heat transfer through microtubes $[25,26,28]$. For the thermal analysis of fluid flow in an electrically heated micro-tube, the solution of conjugate heat transfer (to include effect of the axial conduction through the channel wall) together with Joule heating is required. To properly predict the Joule heating, the electric field within the tube wall has to be obtained.

\subsection{Present study}

An analytic solution is presented for a steady-state conjugate heat transfer in an electrically-heated micro-tube which is a generalized version of the classical Graetz problem. The solution is obtained in the form of integrals by the method of Green's functions for the hydrodynamically fully-developed flow of a constant property fluid in a micro-tube. The effect of the conjugate heat transfer together with the internal energy generation within the micro-tube wall due to Joule heating is analyzed. The current analytical model can predict the fluid temperature for a given wall thickness, wall material and applied voltage across the micro-tube. The effects of the wall thickness and the wall material on the normalized temperature distribution and the effectiveness parameter are discussed. The comparison of the normalized temperature for Joule heating and a spatially uniform heating is also presented. Such a model is beneficial for the researchers and/or engineers who would like to thermally condition fluid in a microtube which can easily be realized as the pre-conditioner of a micro-reactor which would handle different species. To the best of authors' knowledge, an analytical model for such a multi-physics problem has not been developed for the analysis of heat transfer in a micro-tube. 


\section{Temperature equations}

The equations describing the temperature in the circular-tube flow and in the adjacent tube wall are given in this section. The geometry is shown in Fig. 1. The outside wall of the flow channel is heated, and the flow in the tube is fully-developed laminar. The inner radius of the tube is $r_{f}$ and the outer radius of the tube is $r_{w}$. The temperature satisfies the following equations:

wall : $\begin{aligned} & \frac{\partial^{2} T_{w}}{\partial x^{2}}+\frac{1}{r} \frac{\partial}{\partial r}\left(r \frac{\partial T_{w}}{\partial r}\right)+\frac{g_{w}}{k_{w}} \\ = & 0 ; \quad r_{f}<r<r_{w}, \quad-\infty<x<\infty\end{aligned}$

fluid : $\frac{\partial^{2} T_{f}}{\partial x^{2}}+\frac{1}{r} \frac{\partial}{\partial r}\left(r \frac{\partial T_{f}}{\partial r}\right)+\frac{g_{f}}{k_{f}}$

$$
=\frac{u}{\alpha_{f}} \frac{\partial T_{f}}{\partial x} ; \quad 0<r<r_{f}, \quad-\infty<x<\infty
$$

$\left.k_{w} \frac{\partial T_{w}}{\partial r}\right|_{r=r_{w}}=0 ;\left.\quad \frac{\partial T_{f}}{\partial r}\right|_{r=0}=0$

$T(x \rightarrow \pm \infty, r)<M(M$ is finite $)$

At the fluid-wall interface there are two matching conditions:

$T_{w}\left(r_{f}, x\right)=T_{f}\left(r_{f}, x\right)$

$\left.k_{w}\left(\frac{\partial T_{w}}{\partial r}\right)\right|_{r_{f}}=\left.\left(k_{f} \frac{\partial T_{f}}{\partial r}\right)\right|_{r_{f}}$

Heat is added by volume heating through terms $g_{w}$ and $g_{f}$ with units $\mathrm{W} / \mathrm{m}^{3}$, to describe effects in of Joule heating, microwave heating, viscous dissipation, chemical reaction,etc. Results will be presented later for the thermal response to Joule heating in the tube wall. The centerline of the fluid flow is a zero-flux boundary to represent symmetry. The temperature far upstream and downstream $(x \rightarrow \pm \infty)$ will be bounded if heating terms $\left(g_{w}\right.$ and $\left.g_{f}\right)$ are applied over a finite region, which we use to represent a heat exchanger of length $L$. The above differential equations for the temperature will next be recast as integral equations with the method of Green's functions.

\subsection{Solution for temperature}

The geometry described in the previous section involves two regions, the flowing fluid and the adjacent tube wall. Although these regions are quite different, the equations describing the heat transfer in each region differ only by the convection term. That is, the wall equation may be viewed as a special case of the fluid

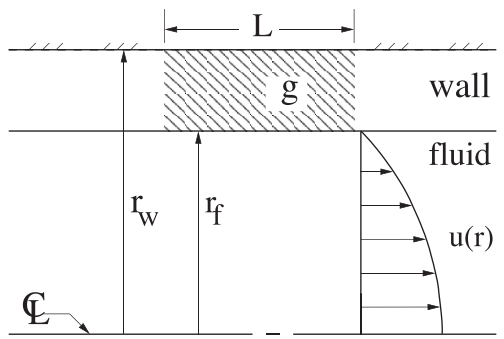

Fig. 1. Geometry of circular microchannel flow heated through tube wall. equation with zero convection velocity. With this observation, the solution will be sought using the method of Green's functions applied to multiple discrete concentric layers within the domain, each of which contains a discrete convection velocity.

The Green's function is a solution to the same equations and boundary conditions as those satisfied by the temperature, except that the distributed heating function $g$ is replaced by a point heat source. Then, the temperature solution is assembled by adding together many Green's functions in such a way that the distributed heat source gis reconstructed from point sources. This adding together takes the form of superposition integrals, as shown below.

Consider layer $i$, a typical layer in the domain with inner radius $r_{i-1}$ and outer radius $r_{i}$ and conductivity $k_{i}$. Let $q_{i-1, i}$ be the heat flux entering layer $i$ from the layer below and let $q_{i+1, i}$ be heat flux that enters layer $i$ from the layer above. Then, the temperature in layer $i$ may be formally stated in the form of integrals with the method of Green's functions, as follows [29, chap. 3]:

$$
\begin{aligned}
T_{i}(x, r)= & \frac{1}{k_{i}} \int_{-\infty}^{\infty} q_{i-1, i}\left(x^{\prime}\right) G_{i}\left(x-x^{\prime}, r, r_{i-1}\right) 2 \pi r_{i-1} d x \prime \\
& +\frac{1}{k_{i}} \int_{-\infty}^{\infty} q_{i+1, i}\left(x^{\prime}\right) G_{i}\left(x-x \prime, r, r_{i}\right) 2 \pi r_{i} d x \prime \\
& +\frac{1}{k_{i}} \int_{-\infty}^{\infty} g_{i}\left(x^{\prime}\right) \int_{r_{i-1}}^{r_{i}} G_{i}(x-x \prime, r, r \prime) 2 \pi r \prime d r \prime d x \prime
\end{aligned}
$$

There are three integral terms, one for each boundary heat flux and one for internal generation $g_{i}$. Internal generation $g_{i}$ is at most a function of position $x$, because we assume that internal heating is spatially uniform across the thickness of layer $i$ (this assumption is consistent with multiple thin layers). Quantity $G_{i}$ is the Green's function (GF) that is evaluated at locations appropriate for each integral term. Assume for the moment that the Green's function is known. Applying Fourier transform, the integrals on $x^{\prime}$ can be stripped away. The Fourier transform is defined by the following transform pair:

$\bar{T}_{i}(r)=\int_{-\infty}^{\infty} T_{i}(x, r) e^{-j \beta x} d x$

$T_{i}(x, r)=\frac{1}{2 \pi} \int_{-\infty}^{\infty} \bar{T}_{i}(r) e^{j \beta x} d \beta$

Apply the Fourier transform to Eq. (2.5)

$$
\begin{aligned}
\bar{T}_{i}(r)= & \frac{1}{k_{i}} \bar{q}_{i-1, i} \bar{G}_{i}\left(r, r_{i-1}\right) 2 \pi r_{i-1} \\
& +\frac{1}{k_{i}} \bar{q}_{i+1, i} \bar{G}_{i}\left(r, r_{i}\right) 2 \pi r_{i} \\
& +\frac{1}{k_{i}} \bar{g}_{i} \int_{r_{i-1}}^{r_{i}} \bar{G}_{i}(r, r \prime) 2 \pi r \prime d r \prime
\end{aligned}
$$

Note that the convolution rule strips away the integrals over $x^{\prime}$. The dependence on wave number $\beta$ has been suppressed to streamline the notation. Quantity $\bar{G}_{i}$, the Fourier-space GF for a typical layer, is given in the Appendix. The above form of the temperature can be used to construct solutions for the heat fluxes across the interfaces between layers as a next step. 


\subsection{Two-layer solution}

To demonstrate the solution method, consider a two-layer geometry composed of a fluid (layer 1 ) and the adjacent wall (layer 2). For this simple example the fluid layer $\left(0<r<r_{1}\right)$ has uniform velocity $u_{1}$ (slug flow) and the wall layer is stationary $\left(u_{2}=0\right)$. There is no internal heating in the fluid $\left(g_{1}=0\right)$. The solution begins by evaluating the temperature in layer 1 (fluid layer) at the fluid-solid interface (location $r_{1}$ )

$\bar{T}_{1}\left(r_{1}\right)=\frac{1}{k_{1}} \bar{q}_{21} \bar{G}_{1}\left(r_{1}, r_{1}\right) 2 \pi r_{1}$

There is only one integral term associated with heat flux at the outer boundary of layer 1 . Next the temperature in layer 2 (wall layer) is evaluated at the fluid-wall interface (at $\left.r_{1}\right)$ :

$\bar{T}_{2}\left(r_{1}\right)=\frac{1}{k_{2}} \bar{q}_{12} \bar{G}_{2}\left(r_{1}, r_{1}\right) 2 \pi r_{1}+\frac{1}{k_{2}} \bar{g}_{2} \bar{G}_{2}\left(r_{1}, r_{2}\right) 2 \pi r_{2}$

In region 2 , there are two contributions to the temperature, one from heat flux at the inner diameter and the other from internal generation. Now, at the fluid-wall interface, there are two matching conditions: the temperatures match; and, the heat flux leaving one layer enters the adjacent layer. In Fourier space these matching conditions may be written:

$\bar{T}_{1}\left(r_{1}\right)=\bar{T}_{2}\left(r_{1}\right)$

$\bar{q}_{21}=-\bar{q}_{12}$

In the four previous equations, the unknowns are $\bar{q}_{12}, \bar{q}_{21}, \bar{T}_{1}$, and $\bar{T}_{2}$. Thus, an algebraic solution may be found by combining the above four equations

$\bar{q}_{21}=\frac{\bar{g}_{2} \bar{G}_{2}\left(r_{1}, r_{2}\right) r_{2} / k_{2}}{\bar{G}_{1}\left(r_{1}, r_{1}\right) r_{1} / k_{1}+\bar{G}_{2}\left(r_{1}, r_{1}\right) r_{1} / k_{2}}$

This is the heat flux passing from the stationary wall to the flowing fluid. Now that the heat flux is known, the temperature in Fourier space in the fluid or wall can be found from Eq. (2.9) or Eq. (2.10), respectively. The temperature in real space must be found from the inverse-Fourier transform defined by Eq. (2.7). The twolayer solution given here is to demonstrate the GF method, however the results given later are computed from a multilayer solution.

\subsection{Multi-layer solution}

The calculation of the heat transfer in the fluid flow and in the adjacent wall is carried out by discretizing the fluid velocity distribution into a collection of concentric layers, each one sliding over its neighbors with piecewise constant velocity (see Fig. 2 for a

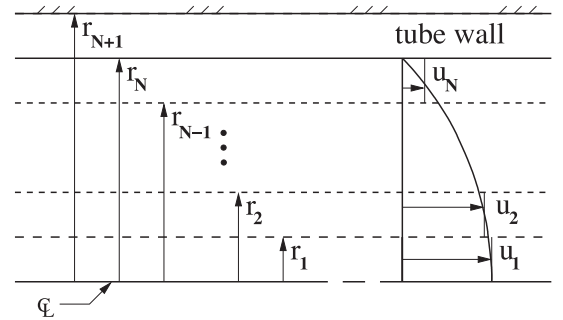

Fig. 2. Multiple concentric layers used to describe the velocity distribution in the circular tube (the tube wall is a layer with zero velocity). schematic of the multilayer for describing the velocity). The solution method begins by evaluating the temperature in each layer at the layer interfaces, as a function of the (initially unknown) interface heat fluxes. Suitable matching conditions at each interface are used to construct a set of algebraic equations for the unknown heat fluxes at each layer interface. The heat fluxes are then found from a matrix solution. Once the heat fluxes are found, the temperature in any layer is given by Eq. (2.5). The matrix equation is given in Appendix C; for a full discussion of the multi-layer approach applied to parallel-plate flow, see Ref. [18].

\subsection{Mean fluid temperature}

An important feature of the layered-fluid approach is that it allows for rapid computer computation of the mean temperature (bulk temperature) in the fluid. In an earlier study with a series solution for the fluid GF, evaluation of the mean temperature would have required additional evaluations of the series, at considerable computational cost.

The mean temperature is defined as a velocity-weighted average temperature in the fluid. For the circular tube, the mean temperature is given in Fourier space by

$\bar{T}_{m}=\frac{1}{U \pi r_{f}^{2}} \int_{r=0}^{r_{f}} u(r) \bar{T}(r) 2 \pi r d r$

where $u(r)$ is the local velocity and $U$ is the average velocity in the circular tube. For the layered description of the fluid used here, the single integral across the tube may be replaced by a sum of integrals over all fluid layers:

$\bar{T}_{m}=\frac{1}{U \pi r_{f}^{2}}\left\{u_{1} \int_{o}^{r_{1}} \bar{T}_{1}(r) 2 \pi r d r+\sum_{i=2}^{N} u_{i} \int_{r_{i-1}}^{r_{i}} \bar{T}_{i}(r) 2 \pi r d r\right\}$

where $T_{i}$ is the temperature and $u_{i}$ is the uniform velocity in layer $i$. If the layers are sufficiently thin, then the integral across each concentric layer may be replaced, to good approximation, with the simple average of the integrand at the two boundaries of each layer. That is,

$\bar{T}_{m}=\frac{1}{U r_{f}^{2}}\left\{u_{1} r_{1}^{2} \bar{T}_{1}\left(r_{1}\right)+\sum_{i=2}^{n} u_{i}\left[r_{i} \bar{T}_{i}\left(r_{i}\right)+r_{i-1} \bar{T}_{i}\left(r_{i-1}\right)\right]\left(r_{i}-r_{i-1}\right)\right\}$

The integral of the first region has been replaced by the temperature at $r_{1}$. These layer-boundary temperatures are important because they may be computed at little cost from already-known quantities.

\section{Joule heating}

In this section, the spatially-varying Joule heating in the metal tube is developed. The tube is heated electrically by the application of an external voltage difference across two circumferential electrodes on the tube outer surface. Refer to Fig. 3. The local volumetric heating $g\left[\mathrm{~W} / \mathrm{m}^{3}\right]$ caused by electrical dissipation in a solid is given by:

$g=\frac{1}{\rho}|\nabla \phi|^{2}=\frac{1}{\rho}\left[\left(\frac{\partial \phi}{\partial r}\right)^{2}+\left(\frac{\partial \phi}{\partial x}\right)^{2}\right]$

where $\rho$ is the resistivity (ohm $\cdot \mathrm{m}$ ) and $\nabla \phi$ is the gradient of the 


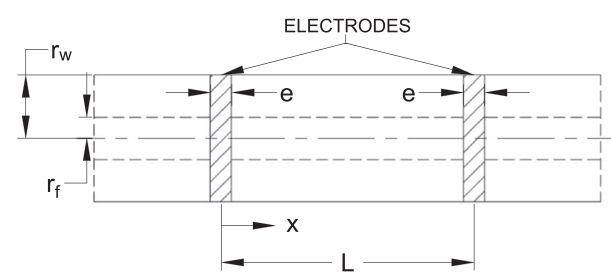

Fig. 3. Geometry of electrodes on tube for resistance heating.

electric potential (volts/m) in the body. The units may be understood with the following identity: 1 Watt $=(\text { volt })^{2} /(\mathrm{ohm})$. In this development, the resistivity is treated as a fixed value throughout the tube wall; that is, the resistivity is uncoupled from the thermal effects. The electric potential satisfies the Laplace equation in a body with externally applied voltage. A full solution requires a mixed-boundary problem, with Type- 1 boundary (Dirichlet type with specified voltage) on the electrodes and Type-2 boundary (Neumann type with derivative equal to zero) elsewhere. To avoid this mixed-type boundary, we have chosen to treat the electrodes as spatially-uniform current sources, a good approximation for small electrodes and for the potential evaluated away from the electrodes. This approach provides for Type-2 (Neumann) boundaries everywhere on the body, for which the method of Green's functions can be applied to find the voltage potential. The source strengths can later be set to give the desired voltage difference across the electrodes.

\subsection{Solution for electric potential}

The voltage potential in the tube satisfies the following boundary value problem:

$\nabla^{2} \phi=0,-x_{0}<x<x_{0} ; r_{f}<r<r_{w}$

at $r=r_{w}, \frac{\partial \phi}{\partial r}=\left\{\begin{array}{cc}1 ; & -e / 2<x<e / 2 \\ -1 ; & L-e / 2<x<L+e / 2 \\ 0 ; & \text { otherwise }\end{array}\right\}$

$\frac{\partial \phi}{\partial n}=0$ on all surfaces

Here, $\partial / \partial n$ is the outward normal derivative on the body surface. The two electrodes are of equal size, one with a positive source and one with a negative source (i.e. a sink) of unit strength. The tube is treated as a finite body on $-x_{0}<x<x_{0}$ which is an approximation to the infinite-domain body of interest. With the method of Green's functions, the solution for $\phi$ is given by:

$\phi(r, x)=\left[\int_{-e / 2}^{e / 2} G\left|r \prime=r_{w} 2 \pi r_{w} d x \prime-\int_{L-e / 2}^{L+e / 2} G\right| r \prime=r_{w} 2 \pi r_{w} d x \prime\right]$

where $G$ is the Green's function for this geometry which is the voltage response to a small current source. In the above expression, the Green's function is integrated to distribute the small sources over the electrodes at $r^{\prime}=r_{w}$. This Green's function is given in Appendix B. Next, the source strength $s_{0}$ is set by evaluating the voltage potential at the center of each electrode and requiring the difference to be $1 \mathrm{~V}$. That is: $s_{0}\left[\phi\left(r_{w}, x_{1}\right)-\phi\left(r_{w}, x_{2}\right)\right]=1$ volt, then

$s_{0}=\frac{1}{\phi\left(r_{w}, x_{1}\right)-\phi\left(r_{w}, x_{2}\right)}$

It is important to note that because the goal is to find the Joule heating, it is not necessary to find the additive constant that is usually needed for a Laplace equation solution subject to Neumann boundary conditions. That is, the voltage difference across the electrodes, not the voltage itself, determines the level of Joule heating.

\subsection{Average heating across the tube radius}

The heating function $g$ depends strongly on $x$ but depends weakly on $r$ for geometries of interest here. Specifically, for long tubes $\left(L / r_{f}=200\right)$ and for radius ratios in the range $2<r_{w} / r_{f}<10$, function $g$ is nearly constant across tube radius $r$, except in a small region near the electrodes. Plots that show radial variation to support this point are given in Ref. [30]. Therefore, for the present work, the function $g(r, x)$ from the development above is averaged over the radius according to:

$g_{a v}(x)=\frac{1}{\pi\left(r_{w}^{2}-r_{f}^{2}\right)} \int_{r_{f}}^{r_{w}} g(r, x) 2 \pi r d r$

It is the radius-averaged heating function that is applied to the combined conduction-convection problem. The volume average heating introduced between the electrodes is given by

$g_{0}=\frac{1}{\left(x_{2}-x_{1}\right) \pi\left(r_{w}^{2}-r_{f}^{2}\right)} \int_{x=-\infty}^{\infty} \int_{r_{f}}^{r_{w}} g(r, x) 2 \pi r d r d x$

This radius-averaged heating function, normalized by the volume average heating, is plotted in Fig. 4 for several tube-wall thicknesses.

\section{Numerical considerations}

Some care was needed to obtain efficient evaluation of the Fourier-inversion integral in Eq. (2.7), which is an improper integral (limits at infinity). Our previous experience with this type of integral was that improper integral on $0<\beta<\infty$ could be replaced by a

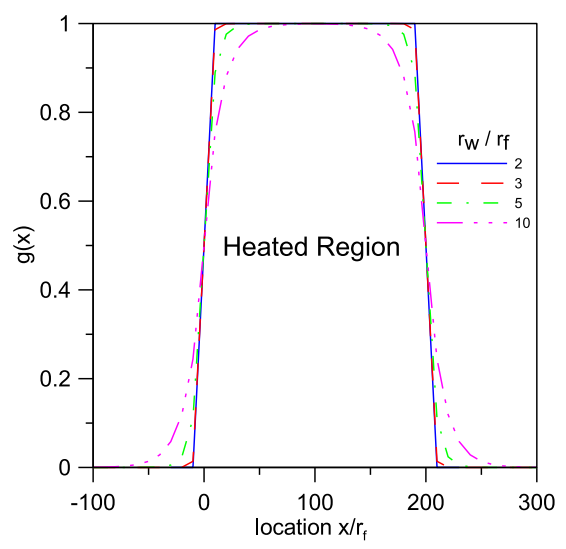

Fig. 4. Normalized heating function $g_{a v}(x) / g_{0}$ for several tube-wall thicknesses for $L / r_{f}=200$. 
summation of proper integrals, each of fixed width and computed by a standard numerical integration scheme, beginning at $\beta=0$. Additional terms of this series were added until the fractional change in the magnitude of the running sum was less than a tolerance to provide 5-digit precision. This series of integrals converges because although the integrand has a single large peak near $\beta=0$, followed by oscillations above and below zero as $\beta$ increases, at $\beta$ values far from zero the integrand decays towards zero. In the present work, however, we found that the use of a fixed-size subinterval for integration sometimes failed when the $\beta$-width of the single large peak was much smaller than the integration subdomain. The shape of the peak depends on the flow parameters or physical location. We addressed this challenge by including a subroutine to examine each integrand to find the width of the peak near $\beta=0$. Then this width was used as the domain size for the subintervals of integration. The additional computer time spent in identifying the width of the peak was more than offset by the improvement in integration efficiency. The integral over $-\infty<\beta<0$ was handled in a similar way. All the coding was carried out with variables of type double-precision complex in Fortran 95.

Another challenge was that for some situations, large values of $\beta$ were required in the inverse-transform integral, which meant that the Bessel functions in the GF were evaluated at large arguments. To avoid numerical overflow in these situations, special exponentially-scaled forms of the Bessel functions for complex arguments were required [31].

\section{Results}

In this section, temperature results are given for heat transfer to water flow in thick-walled circular tubes with electrical-resistance heating introduced through circumferential electrodes on the outer surface. Two tube materials are considered here, nickel with conductivity ratio $k_{w} / k_{f}=143.7$, and copper with conductivity ratio $k_{w} / k_{f}=654.7$.

\subsection{Fluid-bulk temperature in nickel tubes}

Fig. 5 shows the bulk-fluid temperature versus axial location for tubes with electrodes located at $x / r_{f}=0$ and $x / r_{f}=200$ for different Pe. In Fig. 5 by varying the Pe value, we intend to show the effect of various flow rates with fixed values of thermal properties and fixed inner tube radius. By varying $\left(r_{w} / r_{f}\right)$, we intend to show the effect of varying tube-wall thickness at fixed $r_{f}$. The temperature is normalized as:

$T^{+}=\frac{T-T_{0}}{q_{0} r_{f} / k}$ where $q_{0}=\frac{Q}{2 \pi r_{f} L}$

Quantity $q_{0}$ is the equivalent heat flux, as though all of the added heat flow $Q$ (Watts) provided by Joule heating reaches the fluid through the fluid-wall interface area $2 \pi r_{f} L$. This normalization is appropriate for comparing temperature among tubes with different wall thicknesses and different amounts of Joule heating. With this normalization, Fig. 5-(a) shows results for $P e=1.0$, that is, a low fluid-flow rate. The bulk temperature is plotted for four values of the tube-wall thickness, $r_{w} / r_{f}=2.0,3.0,5.0,10$. The bulk temperature rises in the heated region $\left(0<x / r_{f}<200\right)$ but at this low fluid-flow rate the heat transfer is strongly influenced by axial heat conduction in the wall, as evidenced by an anticipatory upstream temperature increase, and by a temperature decrease downstream of the heated region. The effect of wall conduction is stronger as $r_{w} / r_{f}$ increases; the $r_{w} / r_{f}=10$ curve is nearly symmetric around the heated region, indicating that this very thick tube wall is dominated by heat conduction under these conditions.

Fig. 5-(b) shows the bulk temperature under the same conditions as Fig. 5-(a) except that the flow rate is higher at $P e=10$. At this fluid-flow rate the overall temperature values are lower (more convection heat transfer than before), and the effect of convection on the shape of the temperature curves is more pronounced. Fig. 5(c) shows the bulk temperature under the same conditions except that the flow rate is higher yet at $P e=100$. At this high fluid-flow rate the bulk-temperature rise is strictly confined to the heated region for $r_{w} / r_{f}=2.0$, although there is a small amount of upstream conduction at higher $\left(r_{w} / r_{f}\right)$ values. Downstream of the heated region the temperature decreases due to wall conduction as before, but the percentage drop in temperature downstream is roughly proportional to the area available for axial conduction in the tube wall. Again, wall conduction is present in all cases, but it is higher in the thicker-wall cases. Note that the cross-section area available for heat conduction increases as $\left(r_{w}\right)^{2}$, so that the $r_{w} / r_{f}=$ 10 case has $(10 / 2)^{2}=25$ times more area for wall conduction than case $r_{w} / r_{f}=2.0$.

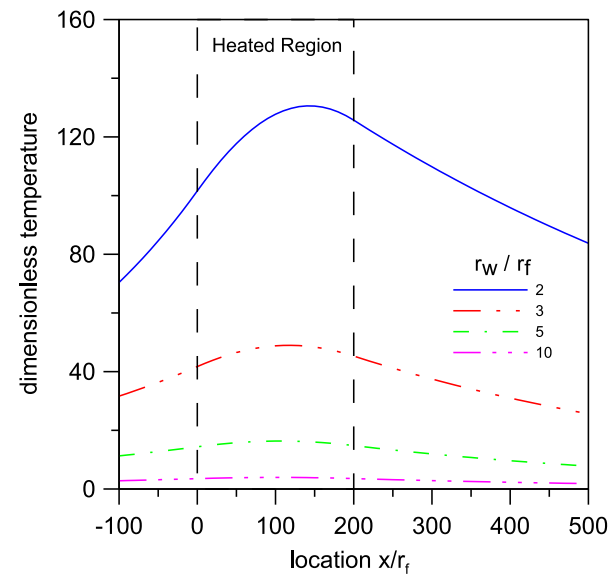

(a) $P e=1.0$

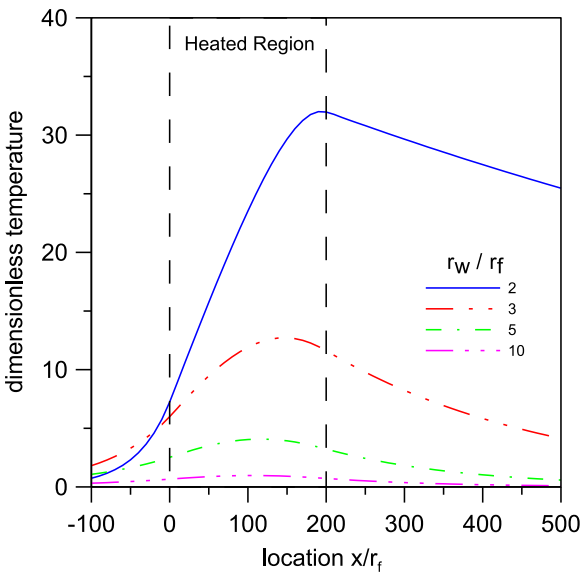

(b) $P e=10$

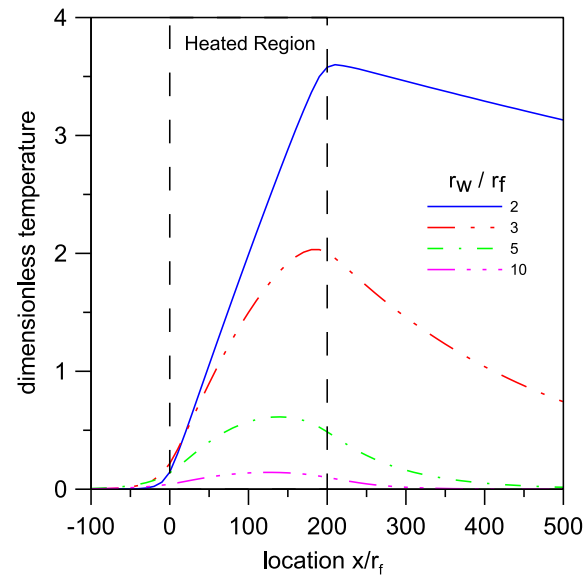

(c) $P e=100$

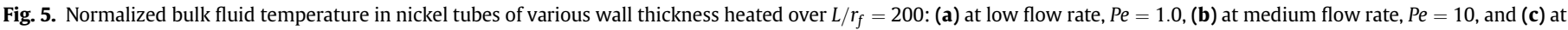
high flow rate, $P e=100$. 


\subsection{Spatial average fluid-bulk temperature}

Temperature results for heated length $L / r_{f}=50$, and for copper tubes are also studied. Rather than provide additional plots of fluidbulk temperature versus $x$, the spatial average bulk temperature over the heated region $(0<x<L)$ has been computed for each case, and these values are plotted in Fig. 6 as a function of tube-wall thickness for several Pe values and for nickel and copper tubes. Fig. 6 shows that the spatial average fluid-bulk temperature decreases as the fluid flow $(\mathrm{Pe})$ increases, which makes sense from the perspective of the microchannel as a heat exchanger. The normalized spatial-average fluid-bulk temperature also decreases as tube size $\left(r_{w} / r_{f}\right)$ increases for all cases, which can be explained by considering that the heated-tube volume increases as $r_{w}$ increases, which then depresses the normalized temperature as it is divided by the amount of heat introduced. In contrast, as the heated length $L$ increases, the temperature increases, as expected from the heat exchanger perspective. The amount of temperature increase is not proportional to length increase, however, as might be expected from a macro-sized heat exchanger, because of the effect of wall

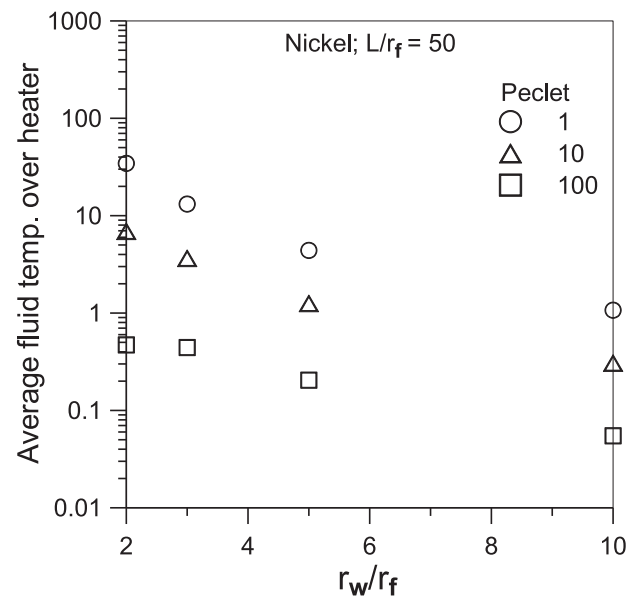

(a)

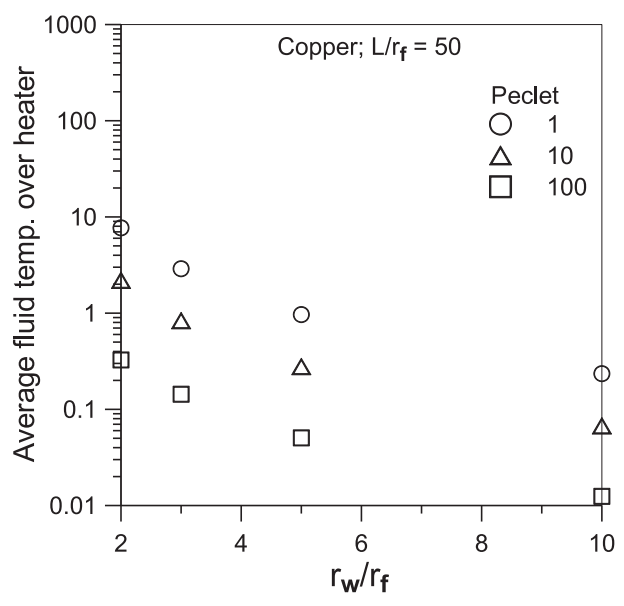

(c) conduction, the effect of which is magnified as tube-wall thickness increases. The data plotted in Fig. 6 is also given in Table 2.

\subsection{Comparison with piecewise-uniform heating}

In this section, the change in temperature is explored for when the electric-potential heating distribution is compared with piecewise-uniform heating over the region $(0<x<L)$. The purpose of this comparison is to identify the circumstances for which the electric-potential calculation, needed to find the precise Jouleheating distribution, may be omitted without sacrificing accuracy in the result. To make this comparison, the bulk-temperature calculation discussed in the previous section was repeated but with spatially uniform heating in the tube. For each comparison, the spatially-uniform heating case introduces the same total power, in Watts, as the corresponding electric-potential-heating case. The two temperatures are compared as follows: the temperature difference between them is normalized by the peak temperature in the electric-potential-heating results. That is, the normalized temperature difference is given by:

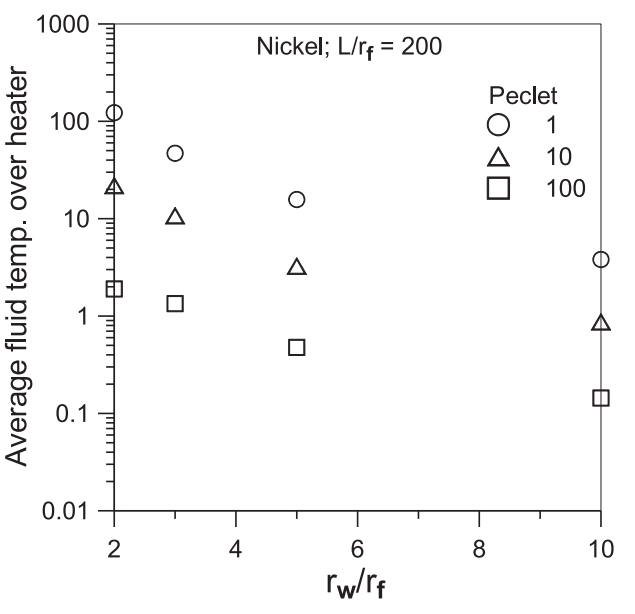

(b)

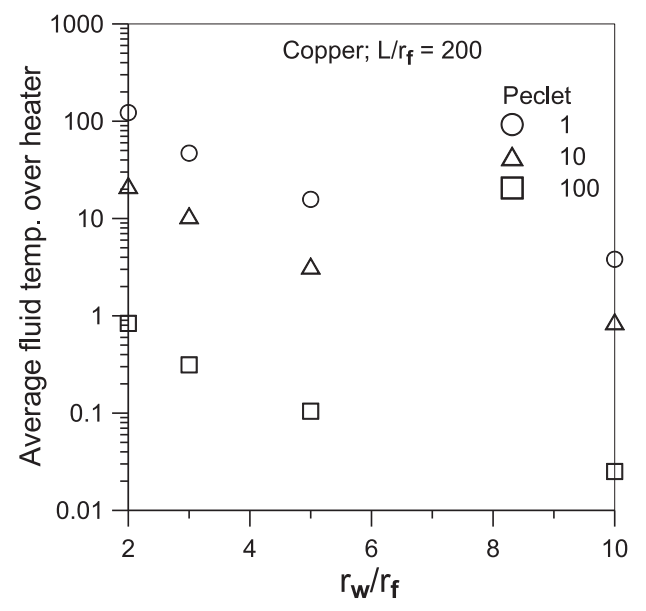

(d)

Fig. 6. Spatial average temperature on heated region, normalized, versus tube-wall thickness, for two wall materials $(\mathrm{Ni}, \mathrm{Cu})$ and two heated lengths $\left(L / r_{f}=50,200\right)$ for three fluidflow rates. 
Table 2

Average fluid temperature over the heated region as a function of tube-wall thickness and fluid flow (Peclet). This data is plotted in Fig. 8.

\begin{tabular}{lllll}
\hline & $\frac{r_{w}}{r_{f}}$ & $\mathrm{Pe}=1$ & $\mathrm{Pe}=10$ & $\mathrm{Pe}=100$ \\
\hline $\mathrm{Cu} ; L / r_{f}=50$ & 2 & 7.70321 & 2.19988 & 0.32628 \\
& 3 & 2.89638 & 0.83677 & 0.14362 \\
$\mathrm{Cu} ; L / r_{f}=200$ & 5 & 0.96617 & 0.27845 & 0.05051 \\
& 10 & 0.23483 & 0.06745 & 0.01248 \\
& 2 & 27.43192 & 6.32837 & 0.83667 \\
$\mathrm{Ni} ; L / r_{f}=50$ & 3 & 10.34180 & 2.38816 & 0.31329 \\
& 5 & 3.45494 & 0.79711 & 0.10463 \\
& 10 & 0.83645 & 0.19183 & 0.02512 \\
$\mathrm{Ni} ; L / r_{f}=200$ & 3 & 34.39058 & 6.91774 & 0.47073 \\
& 5 & 13.14798 & 3.65008 & 0.44275 \\
& 10 & 1.39887 & 1.25630 & 0.20449 \\
& 2 & 122.56180 & 0.30668 & 0.05481 \\
& 3 & 46.94472 & 10.74548 & 1.897810 \\
& 5 & 15.73197 & 3.26015 & 0.47716 \\
& 10 & 3.81076 & 0.87337 & 0.14407 \\
\hline
\end{tabular}

$\Delta T_{\text {norm }}=\Delta T / T_{\text {peak }}$

Fig. 7 shows the normalized temperature difference $\Delta T_{\text {norm }}$ versus position, for the nickel-wall case with $L / r_{f}=200$ for different Pe. Fig. 7-(a) illustrates the case with $P e=1.0$. The plot shows that the difference is a maximum in the middle of the heated region (near $x / r_{f}=100$ ) and is roughly symmetric about this point. At $r_{w} / r_{f}=10$ the difference curve is furthest from zero, and the largest (negative) error is about 0.0042 , less than $1 \%$. The curves for $r_{w} / r_{f}=5.0$ has a (negative) peak error of about $0.001(0.1 \%)$ and the curve for $r_{w} / r_{f}=3.0$ is on the order of $0.0001(0.01 \%)$. Clearly, if $1 \%$ error is acceptable, all these cases at $P e=1.0$ can be treated with simple uniform heating. Fig. 7-(b) shows the normalized temperature difference for the same conditions except now $P e=10$, a medium fluid flow rate. The shape and relative size off the curves in Fig. 7-(b) is similar to those in Fig. 7-(a), but at $P e=10$, the error is roughly three times larger than at $P e=1.0$. Specifically, the peak (negative) error for $r_{w} / r_{f}=10$ is about 0.012 (larger than $1 \%$ ). The error increases as $P e$ increases because convection heat transfer plays a larger role. Previously, we have seen in Fig. 5 that increasing convection introduces a sharper bulk-temperature transition between unheated and heated regions, so it makes sense that, in a comparison between two heating distributions that differ primarily at the edges, increasing convection will emphasize the observed temperature difference. Fig. 7-(c) shows the normalized temperature difference for the same conditions except now $P e=100$, a high fluid flow rate. The previously-noted trend in (negative) error size increasing with increasing Pe continues. Again, the reason is that larger Pe brings larger convective effects. However the shape of the curves are no longer symmetric about the center of the heated region, and, unlike the lower $P e$ cases, there is significant temperature error outside the heated region at $P e=100$.

The above discussion is limited to the nickel tube heated over length $L / r_{f}=200$. Additional comparisons can be performed with tubes heated over length $L / r_{f}=50$ and for copper tubes. For compact presentation of this data, the spatial-average temperature error has been computed for each geometry, and the values plotted in Fig. 8 as a function of $\left(r_{w} / r_{f}\right)$. As $\left(r_{w} / r_{f}\right)$ increases, the error increases for most $\mathrm{Pe}$ values and for both $\mathrm{Cu}$ and $\mathrm{Ni}$ tubes. The exception $P e=1.0$ and Ni tube, but only at $r_{w} / r_{f}>5.0$. One explanation is that for $P e=1.0$, the error averaged only over the heater does not capture the temperature error outside the heated region, which is visible in Fig. 7-(c) (for the nickel wall). Fig. 8 also shows that error is slightly larger for the smaller heated region $\left(L / r_{f}=50\right)$, with a maximum error value about $3 \%$ for the nickel tube and about $2 \%$ for the copper tube, and the maximum error values occur at the highest flow $(P e)$ value. If $1 \%$ error is acceptable, then Fig. 8 shows that piecewise uniform heating is acceptable for the following circumstances: long heated regions $\left(L / r_{f}>50\right)$; thinner tube walls $\left(r_{w} / r_{f} \leq 5.0\right)$; and, lower flow rates (Pe $\left.\leq 10\right)$. For very thick tube walls $\left(r_{w} / r_{f}=10\right)$ and for high flow rates, the full electric-potential description of Joule heated is needed.

\subsection{Heat exchanger effectiveness}

In this section, the performance of the micro-channel is evaluated in terms of heat exchanger effectiveness. The effectiveness of a heat exchanger is defined as the ratio of the actual heat transfer to the fluid divided by maximum possible heat transfer. In the present case the effectiveness takes the form

$\varepsilon=\frac{\dot{m} c_{p}\left(T-T_{0}\right)}{\dot{m} c_{p}\left(T_{\max }-T_{0}\right)}$

Here $T-T_{0}$ is the fluid temperature rise in the heat exchanger, and $\left(T_{\max }-T_{0}\right)$ is the maximum possible fluid temperature rise. For

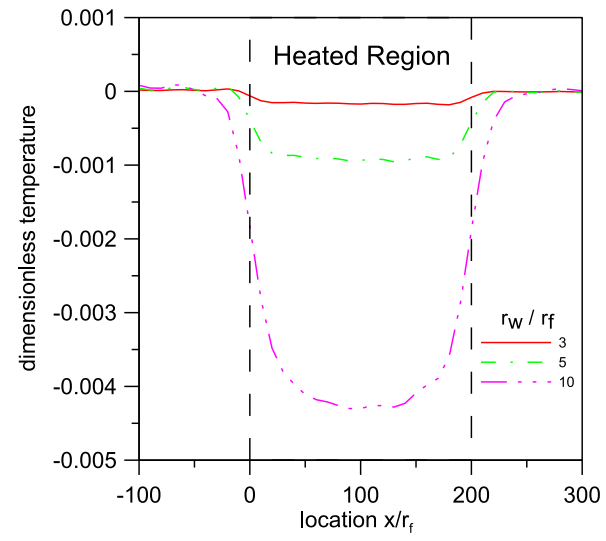

(a) $P e=1.0$

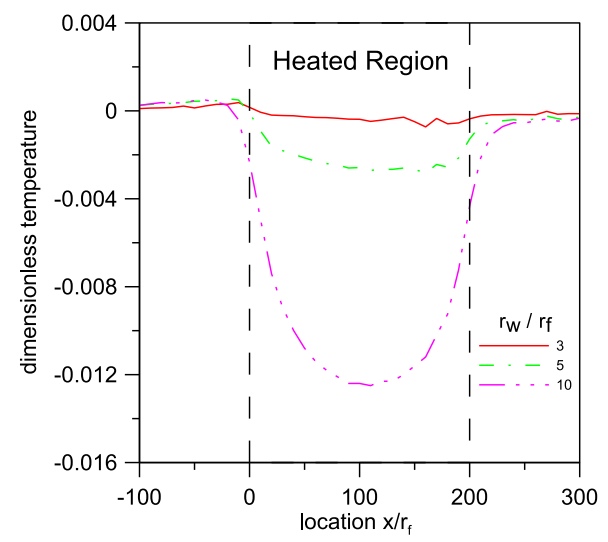

(b) $P e=10$

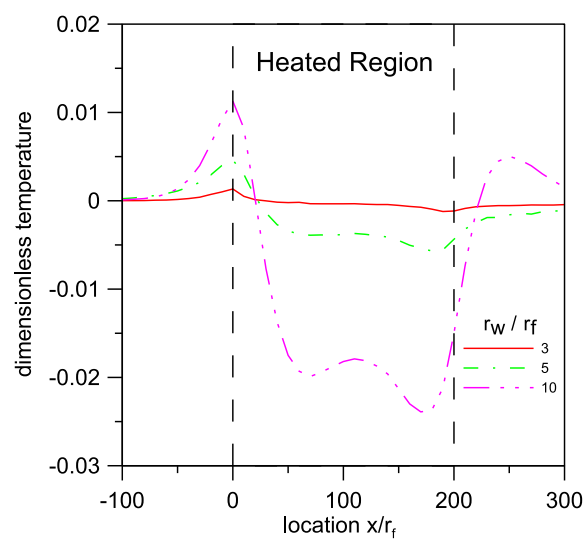

(c) $P e=100$

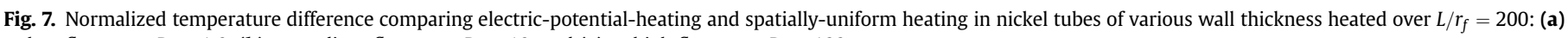
at low flow rate, $P e=1.0$, (b) at medium flow rate, $P e=10$, and (c) at high flow rate, $P e=100$. 


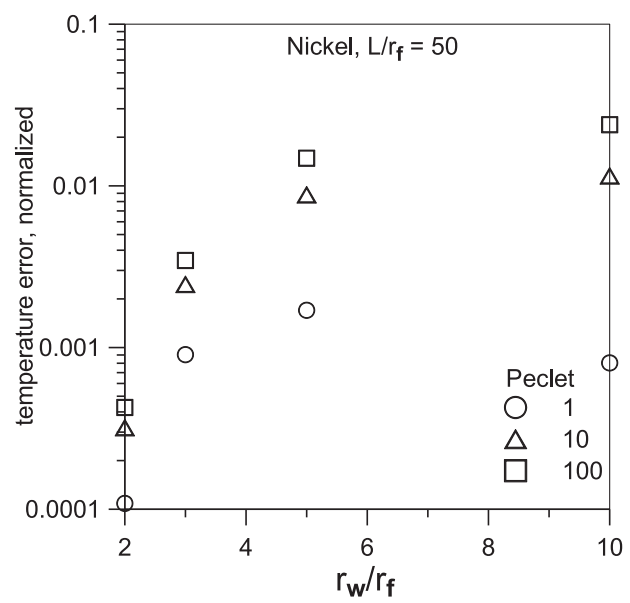

(a)

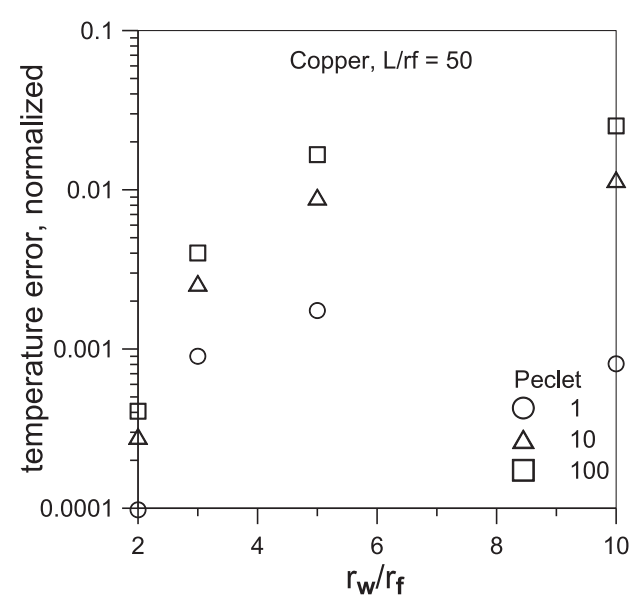

(c)

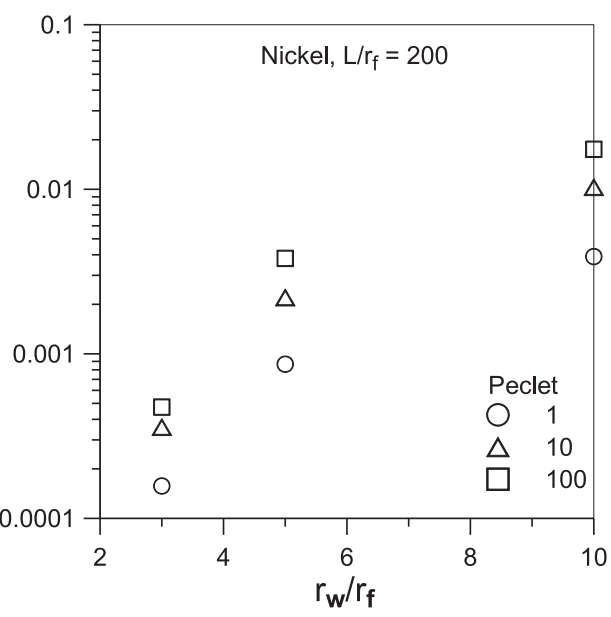

(b)

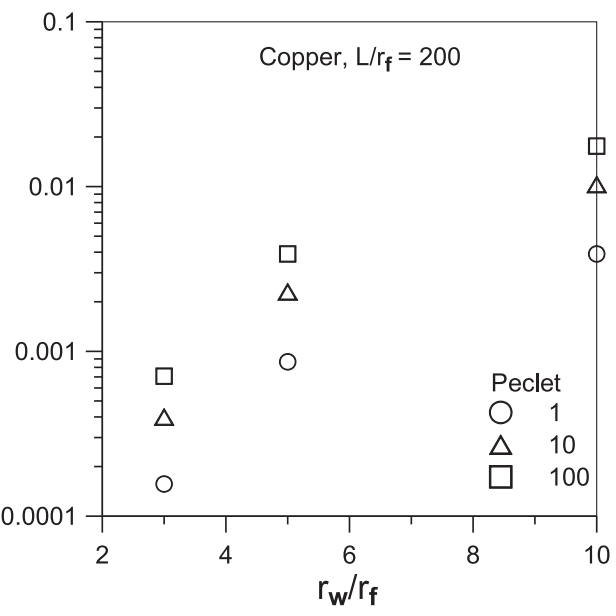

(d)

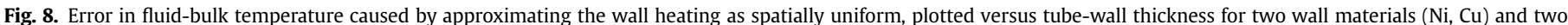
heated lengths $\left(L / r_{f}=50,200\right)$ for three fluid-flow rates.

the present discussion, the spatial average fluid-bulk temperature discussed above is taken as a measure of the actual temperature rise. This is appropriate in the present work because the peak fluid temperature does not occur at the end of the heated region when wall conduction is dominant; the spatial average allows for a reasonable comparison among flows with varying amounts of wall conduction. The maximum possible temperature in the fluid is that fluid temperature that would be achieved if all of the introduced heat enters the fluid. That is, the maximum possible temperature rise in the fluid is given by:

$T_{\max }-T_{0}=\frac{Q}{\dot{m} c_{p}}$

By combining the earlier definition of normalized temperature with the definition of effectiveness, it is possible to show a relationship between the normalized temperature $T^{+}$and heat exchanger effectiveness $\varepsilon$ to be

$\varepsilon=T_{a v e}^{+} \cdot \mathrm{Pe} \cdot \frac{r_{f}}{2 L}$
With the above relationship, the temperature data discussed earlier has been recast as heat exchanger effectiveness, and plotted in Fig. 9. Fig. 9 shows that the effectiveness decreases as $r_{w} / r_{f}$ increases, which means that as wall thickness increases, less of the introduced heat reaches the fluid in the region between the electrodes. Generally the effectiveness increases with $P e$, which is consistent with the expectation that heat transfer increases with fluid flow rate. Effectiveness decreases slightly as heated length increases from 50 to 200, with the decrease ranging for the copperwalled tubes from $11 \%$ at $P e=1.0$ up to $50 \%$ at $P e=100$. From macro-flow theory, short heat exchangers are expected to have higher effectiveness, however the presence of wall conduction is the explanation for the somewhat muted response here. There is a strong effect of wall material on the effectiveness, in proportion to the thermal conductivity at small Pe. Specifically, the effectiveness is lower by a factor of 4.5 for copper compared to nickel at $P e=1.0$; note that copper has higher thermal conductivity than nickel by precisely this factor. This is a clear demonstration that at $P e=1.0$, the heat transfer is dominated by wall conduction. 


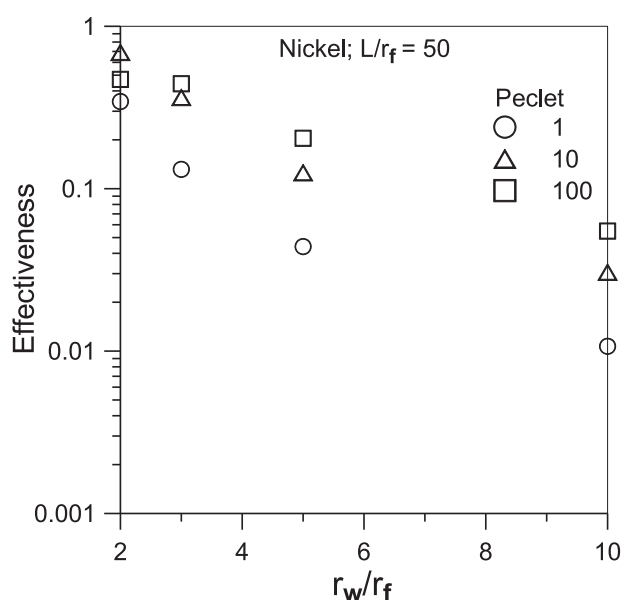

(a)

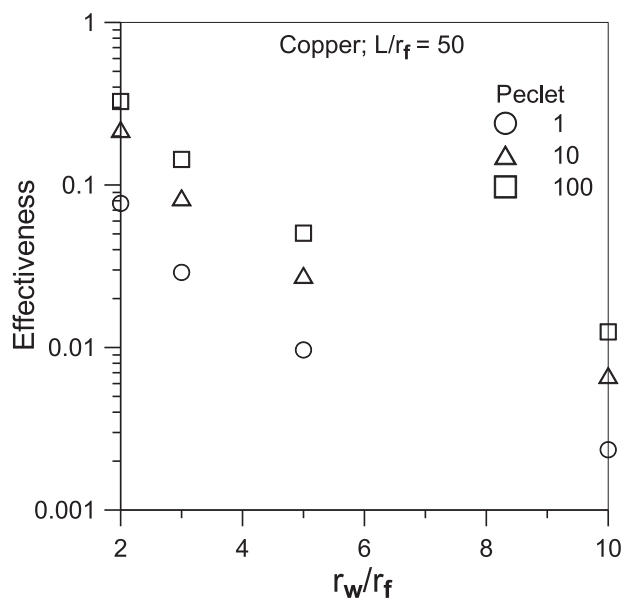

(c)

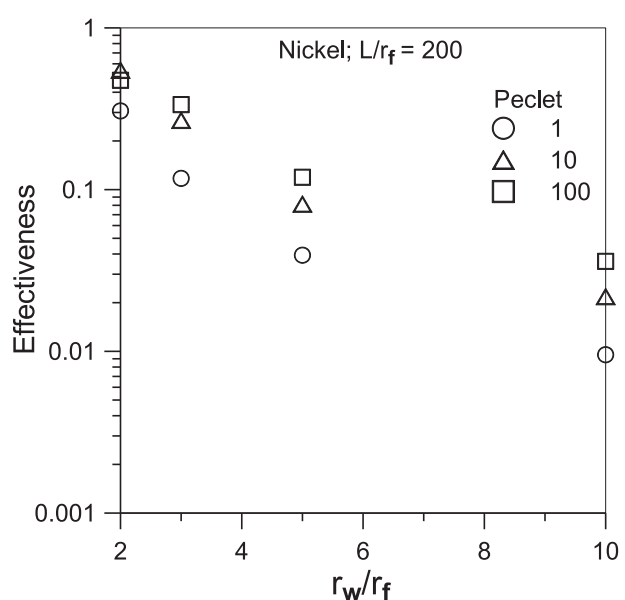

(b)

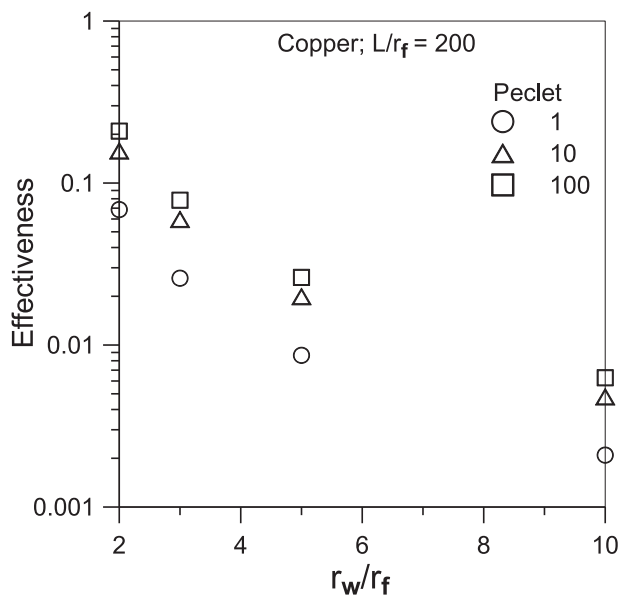

(d)

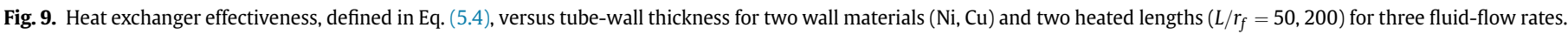

\section{Summary}

Modeling of a thick-walled circular micro-tube heated by electrical current passing through the tube wall with water flow inside the tube is described in this paper. The spatial distribution of Joule heating in the tube wall is found analytically from the electric potential distribution between circumferential electrodes on the tube surface. The energy equations for the fluid and the wall, which include the effect of heat diffusion in the axial direction, are solved analytically with Green's functions. The analytical solution has the form of integrals from which numerical results are obtained by numerical integration for water flow in metal tubes. Fluid-bulk temperature in the tube are computed as a function of tube-wall thickness, fluid flow rate, wall material, and electrode spacing. Fluid-bulk temperature results are presented as a function of location along the tube under several conditions, and the spatialaverage temperature rise over the heated region is used to provide an overview of the behavior over all conditions studied. The results show that higher fluid-outlet temperatures are obtained under the following conditions: low fluid-flow rate, thin tube wall, low tube-wall thermal conductivity, and wide spacing between electrodes, as expected.

By viewing the micro-tube as a heat exchanger, the heat exchange exchanger effectiveness is computed to show that the tubewall thickness and tube-wall thermal conductivity have a strong impact, with lesser impact of fluid-flow rate and heat exchanger length. The most effective heat exchanger, in the form of an electrically-heated micro-tube, has a thin tube wall with low thermal conductivity, and a high fluid-flow rate. A study of the Joule heating is included, to show the conditions under which the spatially-varying heating, computed from electric-potential theory, may be replaced by simple uniform heating in the tube wall. For error less than one percent, the uniform heating condition is acceptable for longer heated regions $\left(L / r_{f}>50\right)$, thinner tube walls $\left(r_{w} / r_{f} \leq 5\right)$, and lower fluid-flow rates (Pe $\left.\leq 10\right)$. The findings of this study will be beneficial for the scientists and engineers who would like to design micro-heat exchangers, micro-reactors, and microfluidic fuel cells. 


\section{Acknowledgments}

Support from the National Science Foundation is greatly acknowledged from grant CBET-1250626 under program manager S. Acharya. Thanks are extended to Nebraska undergraduate Kyle Ellison who ran computer codes and plotted graphs for this project.

\section{Appendix A. Green's function for heat transfer}

In this Appendix the GF is given for steady temperature in a slug flow in a circular-tube annulus $(a<r<b)$. This GF, denoted $G\left(x-x^{\prime}, r, r \prime\right)$, is the steady response at location $(x, r)$ caused by unit-amplitude steady heating introduced at point $(x \prime, r \prime)$. The GF satisfies the following differential equations (subscript $i$ has been suppressed to simplify the notation)

$\frac{\partial^{2} G}{\partial x^{2}}+\frac{1}{r} \frac{\partial}{\partial r}\left(r \frac{\partial G}{\partial r}\right)-\frac{u}{\alpha} \frac{\partial G}{\partial x}=-\delta(x-x \prime) \frac{\delta(r-r \prime)}{2 \pi r^{\prime}} ;$

Here $u$ is the spatially-uniform velocity in the $x$-direction. The boundary conditions are

At $\mathrm{x} \rightarrow \pm \infty, \quad G$ is bounded.

At $r=a, \quad \frac{\partial G}{\partial r}=0$

At $r=b, \quad \frac{\partial G}{\partial r}=0$

Note that the boundaries at $(r=a)$ and at $(r=b)$ are of the second kind. For the present work, the spatial-Fourier transform of this GF is needed. First use a simple change of variable to replace $\left(x-x^{\prime}\right)$ by $x$. Then apply the Fourier transform, Eq. [13], to the above differential equation, to find

$\frac{1}{r} \frac{\partial}{\partial r}\left(r \frac{\partial \bar{G}}{\partial r}\right)-\nu^{2} \bar{G}=-\frac{\delta(r-r \prime)}{2 \pi r \prime}$

where $\nu^{2}=\beta^{2}+j \beta u / \alpha$. The solution for this GF in Fourier space is given by (see Ref. [29], chap. 9, or [32])

$\bar{G}(r \mid r \prime)=\frac{1}{2 \pi\left(1-A_{1} A_{2}\right)} \begin{cases}{\left[A_{2} I_{0}(\nu r \prime)+K_{0}(\nu r \prime)\right]\left[I_{0}(\nu r)+A_{1} K_{0}(\nu r)\right],} & r<r \\ {\left[A_{2} I_{0}(\nu r)+K_{0}(\nu r)\right]\left[I_{0}(\nu r \prime)+A_{1} K_{0}(\nu r \prime)\right],} & r<r\end{cases}$

$A_{1}=\frac{I_{1}(\nu a)}{K_{1}(\nu a)}, A_{2}=\frac{K_{1}(\nu b)}{I_{1}(\nu b)}$

Note that in the special case $a=0$, the layer takes the shape of a small cylindrical region centered at the origin, and the GF is simplified by $A_{1}=0$. Although this GF was defined for uniform flow, the stationary wall is also described by this GF for the special case $u=0$ (zero velocity). This form of the GF is used in all the results presented in this paper.

The spatial integral of the GF over the annular layer is needed when internal generation is present. The spatial integral of the GF is defined by
$\overline{\bar{G}}(r)=\int_{b}^{a} \bar{G}(r, r \prime) 2 \pi r / d r \prime$

For use in the matrix solution, the spatial integral of the GF is evaluated either at the inner radius or the outer radius, and these values are given by

$$
\begin{aligned}
& \overline{\bar{G}}(a)=\frac{1}{\nu\left(1-A_{1} A_{2}\right)}\left[A_{2} N+M\right] \times\left[I_{0}(\nu a)+A_{1} K_{0}(\nu a)\right] \\
& \overline{\bar{G}}(b)=\frac{1}{\nu\left(1-A_{1} A_{2}\right)}\left[A_{2} I_{0}(\nu b)+K_{0}(\nu b)\right] \times\left[N+A_{1} M\right] \\
& \text { where } N=b I_{1}(\nu b)-a I_{1}(\nu a) \\
& M=a K_{1}(\nu a)-b K_{1}(\nu b)
\end{aligned}
$$

\section{Appendix B. Green's function for electric potential}

In this Appendix the Green's function (GF) for the electric potential in the tube wall is given. The GF satisfies

$\frac{\partial^{2} G}{\partial x^{2}}+\frac{1}{r} \frac{\partial}{\partial r}\left(r \frac{\partial G}{\partial r}\right)=-\delta(x-x \prime) \frac{\delta(r-r \prime)}{2 \pi r^{\prime}} ; \quad\left\{\begin{array}{l}-x_{0}<x<+x_{0} \\ a<r<b\end{array}\right.$

When all of the boundary conditions are homogeneous of the Neumann type (zero slope), the Green's function is given by 30

$$
\begin{array}{cc}
G\left(x, r \mid x^{\prime}, b\right)= & Q_{0}(r, b) \\
& +\frac{1}{2} \sum_{p=1}^{\infty} \cos \left(\eta_{p}\left(x+x_{0}\right)\right) \cos \left(\eta_{p}\left(x^{\prime}+x_{0}\right)\right) Q_{p}(r, b)
\end{array}
$$

where

The finite domain given here $\left(-x_{0}<x<+x_{0}\right)$, which was chosen for ease of computation, is different than the infinite-length tube for the heat-transfer problem discussed in the body of the paper. 
However the computed value of the generation term $g$, found using two electrodes and the relation for Joule heating (Eq. (3.1)), is completely compatible with the heat transfer problem, as long as the electrodes are inset a sufficient distance from the ends of the finite-length tube. The reason is that the Joule heating tends to zero outside of the electrodes over a distance on the order of length $(b-a)$. As long as the electrode-inset distance is substantially larger than $(b-a)$, then the electric-potential result is independent of tube length $x_{0}$. To be clear, the finite-tube GF described here is used only for the electric potential calculation; the heat transfer problem discussed in the body of the paper is carried on the infinite-tube domain using the GF described in Appendix A.

\section{Appendix C. Matrix equation for multilayer solution}

The layered geometry shown in Fig. 4 has $N+1$ concentric layers, numbered from 1 to $N+1$, with $N$ interfaces between the layers. Layer $N+1$ is the wall with zero velocity. Layers 1 through $N$ are located in the laminar flow, with uniform velocity in each layer set to a value to produce a piecewise version of the laminar parabolic velocity distribution. Within layer $i$, the interfaces are at coordinates $r_{i}$ and $r_{i-1}$. Layer $i$ has thickness $\left(r_{i}-r_{i-1}\right)$ and thermal conductivity $k_{i}$. At the interfaces between the layers, let $q_{n m}$ represent the heat flux leaving layer $n$ and entering layer $m$. In the formulation given below, heating is primarily caused by external heat at the outside of the wall (layer $N+1$ ), however heat may also be introduced internally within each layer through generation term $\bar{g}_{i}$.

The result is a set of $N$ linear algebraic equations for the unknown heat fluxes, which may be stated in matrix form:

$$
\begin{aligned}
& {\left[\begin{array}{ccccc}
F_{1}+C_{2} & -D_{2} & 0 & \ldots & 0 \\
-E_{2} & F_{2}+C_{3} & -D_{3} & \ldots & 0 \\
0 & -E_{3} & F_{3}+C_{4} & \ldots & 0 \\
\vdots & \vdots & \vdots & \ddots & -D_{N} \\
0 & 0 & \cdots & -E_{N} & F_{N}+C_{N+1}
\end{array}\right] \times\left[\begin{array}{l}
\bar{q}_{21} \\
\bar{q}_{32} \\
\bar{q}_{43} \\
\vdots \\
\bar{q}_{N+1, N}
\end{array}\right]} \\
& =\left[\begin{array}{l}
\phi_{2} \bar{g}_{2}-\psi_{1} \bar{g}_{1} \\
\phi_{3} \bar{g}_{3}-\psi_{2} \bar{g}_{2} \\
\phi_{4} \bar{g}_{4}-\psi_{3} \bar{g}_{3} \\
\vdots \\
\phi_{N+1} \bar{g}_{N+1}-\psi_{N} \bar{g}_{N}+\bar{p} D_{N+1}
\end{array}\right]
\end{aligned}
$$

The symbols used in the above expression are given below:

$$
\begin{aligned}
C_{i} & =\frac{1}{k_{i}} \bar{G}_{i}\left(r_{i-1}, r_{i-1}\right) 2 \pi r_{i-1} \\
D_{i} & =\frac{1}{k_{i}} \bar{G}_{i}\left(r_{i-1}, r_{i}\right) 2 \pi r_{i} \\
E_{i} & =\frac{1}{k_{i}} \bar{G}_{i}\left(r_{i}, r_{i-1}\right) 2 \pi r_{i-1} \\
F_{i} & =\frac{1}{k_{i}} \bar{G}_{i}\left(r_{i}, r_{i}\right) 2 \pi r_{i} \\
\phi_{i} & =\frac{1}{k_{i}} \overline{\bar{G}}_{i}\left(r_{i-1}\right) \\
\psi_{i} & =\frac{1}{k_{i}} \overline{\bar{G}}_{i}\left(r_{i}\right)
\end{aligned}
$$

For any multilayered system, this matrix equation may be solved for $N$ unknown heat fluxes $\left(\bar{q}_{i j}\right)$ through all interfaces in the system. Once the heat fluxes are found, the temperature in any layer may be found with Eq. (2.5).

\section{References}

[1] Khan Mesbah G, Fartaj Amir. A review on microchannel heat exchangers and potential applications. Int J Energy Res 2011;35(7):553-82.

[2] Morini Gian Luca. Scaling effects for liquid flows in microchannels. Heat Transf Eng 2006:27(4):64-73.

[3] Beskok A, Karniadakis GE. A model for flows in channels, pipes, and ducts at micro and nano scales. Microscale Thermophys Eng 1999;3:43-77.

[4] Cetin B, Yazicioglu AG, Kakac S. Fluid flow in microtubes with axial conduction including rarefaction and viscous dissipation. Int Comm Heat Mass Transf 2008;35:535-44

[5] Cetin B, Bayer O. Evaluation of Nusselt number for a flow in a microtube using second-order slip model. Therm Sci 2011:15(Suppl. 1):103-9.

[6] Cetin B. Effect of thermal creep on heat transfer for a two-dimensional microchannel flow: an analytical approach. J Heat Transf 2011;135(10): 101070.

[7] Tso CP, Mahulikar SP. The use of the brinkman number for single phase forced convective heat transfer in microchannels. Int J Heat Mass Transf 1998;41(12): 1759-69.

[8] Cetin B, Yazicioglu AG, Kakac S. Slip-flow heat transfer in microtubes with axial conduction and viscous dissipation-An extended Graetz problem. Int J Therm Sci 2009;48:1673-8.

[9] Weilin Qu, Mohiuddin Mala Gh, Dongqing Li. Pressure-driven water flows in trapezoidal silicon microchannels. Int J Heat Mass Transf 2000;43(3):353-64.

[10] Li Dongqing. Electro-viscous effects on pressure-driven liquid flow in microchannels. Colloids Surfaces A Physicochem Eng Aspects 2001;195(1-3): $35-57$.

[11] London AL, Shah RK. Laminar flow forced convection in ducts: a source book for compact heat exchanger analytical data. Academic Press; 1978. p. 78-138.

[12] James Davis E, Gill William N. The effects of axial conduction in the wall on heat transfer with laminar flow. Int J Heat Mass Transf 1970;13(3):459-70.

[13] Mori S, Kawamura Y, Tanimoto A. Conjugated heat transfer to laminar flow with internal heat source in a parallel plate channel. Can J Chem Eng 1979;57: 698-703.

[14] Kroeker CJ, Soliman HM, Ormiston SJ. Three-dimensional thermal analysis of heat sinks with circular cooling micro-channels. Int J Heat Mass Transf 2004;47(22):4733-44.

[15] Nonino C, Savino S, Del Giudice S, Mansutti L. Conjugate forced convection and heat conduction in circular microchannels. Int J Heat Fluid Flow 2009;30(5):823-30.

[16] Sen Soner, Darici Selcuk. Transient conjugate heat transfer in a circular microchannel involving rarefaction, viscous dissipation and axial conduction effects. Appl Therm Eng 2017;111:855-62.

[17] Maranzana Gael, Perry Isabelle, Maillet Denis. Mini- and micro-channels: influence of axial conduction in the walls. Int J Heat Mass Transf 2004;47(17-18):3993-4004.

[18] Cole Kevin D, Çetin Barbaros. The effect of axial conduction on heat transfer in a liquid microchannel flow. Int J Heat Mass Transf 2011;54(11-12):2542-9.

[19] Cole Kevin D, Cetin Barbaros, Brettmann Lukas. Microchannel heat transfer with slip flow and wall effects. J Thermophys Heat Transf 2014;28(3):455-62.

[20] Kabar Yassine, Bessah Rachid, Rebay Mourad. Conjugate heat transfer with rarefaction in parallel plates microchannel. Superlattices Microstruct 2013;60: $370-88$.

[21] Li J, Peterson GP, Cheng P. Three-dimensional analysis of heat transfer in a micro-heat sink with single phase flow. Int J Heat Mass Transf 2004;47(1920): 4215-31.

[22] Kosar Ali. Effect of substrate thickness and material on heat transfer in microchannel heat sinks. Int J.Thermal Sci 2010;49(4):635-42.

[23] Knupp Diego C, Cotta Renato M, Naveira-Cotta Carolina P, Kakac Sadik Transient conjugated heat transfer in microchannels: integral transforms with single domain formulation. Int J Therm Sci 2015;88:248-57.

[24] Duryodhan VS, Singh SG, Agrawal Amit. Heat rate distribution in converging and diverging microchannel in presence of conjugate effect. Int J Heat Mass Transf 2017; 104:1022-33.

[25] Yang Yahui, Hong Chungpyo, Luca Morini Gian, Asako Yutaka. Experimental and numerical investigation of forced convection of subsonic gas flows in microtubes. Int J Heat Mass Transf 2014;78:732-40.

[26] Yang Ahui, Chalabi Habib, Lorenzini Marco, Morini Gian Luca. The effect on the nusselt number of the nonlinear axial temperature distribution of gas flows through microtubes. Heat Transf Eng 2015;35(2):159-70.

[27] Goodfellow company-nickel tube material information. www.goodfellow. com/E/Nickel-Tube.html. Accessed November 25, 2016.

[28] Kaya A, Demiryürek R, Armağan E, Ozaydin-Ince G, Sezen M, Kosar A. Boiling heat transfer enhancement in mini/microtubes via polyhydroxyethylmethacrylate (phema) coatings on inner microtube walls at high 
mass fluxes. J Micromech Microeng 2013;23:115017.

[29] Cole KD, Beck JV, Haji-Sheikh A, Litkouhi B. Heat conduction using Green's functions. 2 edition. New York: Taylor and Francis; 2011.

[30] Finite-length hollow cylinder with neumann boundaries with heat added and removed on circumferential regions on the outer diameter. Exact Analytical
Conduction Toolbox, exact.unl.edu. Accessed: March 03, 2017.

31] Amos DE, Daniel SL. Amoslib special function library. Technical report, NTIS Technical Report 7503, Sandia Laboratory Contract AT(29-1)-789. 081979.

[32] Cole Kevin D, Crittenden Paul E. Steady-periodic heating of a cylinder. J Heat Transf 2009;131(9). 091301-091301. 